International Journal of Biological Sciences

ISSN 1449-2288 www.biolsci.org 2006 2(2):38-47

Review

C2006 Ivyspring International Publisher. All rights reserved

\title{
Retinoic acid signaling and the evolution of chordates
}

\author{
Ferdinand Marlétaz ${ }^{1}$, Linda Z. Holland ${ }^{2}$, Vincent Laudet ${ }^{1}$ and Michael Schubert ${ }^{1}$
}

1. Laboratoire de Biologie Moléculaire de la Cellule, CNRS UMR5161/INRA 1237/ENS Lyon, IFR128 BioSciences/LyonGerland, Ecole Normale Supérieure de Lyon, 46 Allée d'Italie, 69364 Lyon Cedex 07, France

2. Marine Biology Research Division, Scripps Institution of Oceanography, University of California San Diego, La Jolla, CA 92093-0202, USA

Corresponding address: V. Laudet, Laboratoire de Biologie Moléculaire de la Cellule, CNRS UMR5161/INRA 1237/ENS Lyon, IFR128 BioSciences/Lyon-Gerland, Ecole Normale Supérieure de Lyon, 46 Allée d'Italie, 69364 Lyon Cedex 07, France. E-mail: Vincent.Laudet@ens-lyon.fr - Tel: ++33472728190 - Fax: ++33472728080

Received: 2006.02.13; Accepted: 2006.03.15; Published: 2006.04.10

In chordates, which comprise urochordates, cephalochordates and vertebrates, the vitamin A-derived morphogen retinoic acid (RA) has a pivotal role during development. Altering levels of endogenous RA signaling during early embryology leads to severe malformations, mainly due to incorrect positional codes specifying the embryonic anteroposterior body axis. In this review, we present our current understanding of the RA signaling pathway and its roles during chordate development. In particular, we focus on the conserved roles of RA and its downstream mediators, the Hox genes, in conveying positional patterning information to different embryonic tissues, such as the endoderm and the central nervous system. We find that some of the control mechanisms governing RA-mediated patterning are well conserved between vertebrates and invertebrate chordates, such as the cephalochordate amphioxus. In contrast, outside the chordates, evidence for roles of RA signaling is scarce and the evolutionary origin of the RA pathway itself thus remains elusive. In sum, to fully understand the evolutionary history of the RA pathway, future research should focus on identification and study of components of the RA signaling cascade in non-chordate deuterostomes (such as hemichordates and echinoderms) and other invertebrates, such as insects, mollusks and cnidarians.

Key words: amphioxus, anteroposterior patterning, Branchiostoma, deuterostomes, Hox, invertebrate-to-vertebrate transition, phylogeny

\section{Introduction}

Among bilaterian animals, the deuterostomes include the hemichordates, the echinoderms and the chordates. The chordates can further be subdivided into urochordates (=tunicates), cephalochordates and vertebrates (Fig. 1A). Since human beings have often wondered about their own origins, it is not surprising that there have been many studies aimed at understanding the evolutionary history of chordates (i.e. the origin of chordates and the diversification of vertebrates). The main characters that evolved at the base of the chordates are a notochord and a dorsal hollow nerve cord [1], while definite neural crest cells and placodes arose at the base of the vertebrates [2]. However, the evolutionary origins of these structures have been controversial, mainly because of difficulties in determining homologies between chordate-specific characters and structures in non-chordate deuterostomes (i.e. in hemichordates and echinoderms) as well as between the vertebratespecific characters and structures in invertebrate chordates.

The development of many chordate- and vertebrate-specific characters is controlled, directly or indirectly, by retinoic acid (RA), a vitamin A-derived morphogen. In chordate embryos, too much or too little RA during early embryogenesis causes malformations, which are mainly due to a mispatterning of the embryo along the anteroposterior body axis. RA signaling is mediated by $R A$ binding to retinoic acid receptors (RARs), which form heterodimers with retinoid $X$ receptors (RXRs) [3]. During chordate development, important functions of RA signaling are mediated by Hox genes, at least some of which are direct targets of RA [4-6]. In many animals, including most deuterostomes, Hox genes mediate anteroposterior positional patterning of the embryo [7, 8]. Moreover, in vertebrates, Hox genes have been suggested to play important roles in the patterning of all embryonic tissue layers [7,9].

Ever since it became clear that the genetic mechanisms controlling key features of embryonic development are highly conserved between organisms as diverse as fruit flies and mice, comparisons of the developmental mechanisms in different organisms have been used to address questions about animal evolution, particularly about the invertebrate-to-vertebrate transition in chordates [10]. Using this so-called "evo-devo" approach, this review discusses the developmental roles of RA signaling among chordates in regard to chordate origins and vertebrate evolution. The focus is on the roles of RA- and Hox-dependent patterning during development and their conservation during chordate 
evolution. We show that some of the control mechanisms governing RA-mediated patterning are quite conserved among vertebrates and between vertebrates and invertebrate chordates, such as the cephalochordate amphioxus. In contrast, evidence for roles of RA signaling outside the chordates and the evolutionary origin of RA signaling itself still remain elusive.

Figure 1. Deuterostome phylogeny and components of the RA signaling pathway in deuterostomes. A) Deuterostome phylogeny. Echinoderms and hemichordates together establish the sister group of chordates. The urochordates (=tunicates) include ascidians and appendicularians. Urochordates and cephalochordates are invertebrate chordates. The vertebrates include agnathan groups (hagfish and lampreys) as well as the gnathostome chondrichthyans (cartilaginous fish), actinopterygians (ray-finned fish) and sarcopterygians (lobe-finned fish and tetrapods). Within chordates, the phylogenetic relationships between cephalochordates and urochordates and between hagfish and lampreys are still disputed and their respective positions within the tree are thus shown as polytomies. Two important events during deuterostome evolution are the origin of chordates and the origin of vertebrates. The two rounds of extensive gene duplications early during vertebrate diversification are highlighted with green boxes labeled 'R'. B) Components of the RA pathway in deuterostomes. Deuterostome groups, for which a whole genome sequencing (WGS) project has already been finished are indicated with a turquoise "+", those, for which a WGS project is underway, are marked with a turquoise " $(+)$ ". If known, the exact number of $R A R$, Raldh and Cyp26 genes in the genome of a specific deuterostome group is indicated, the certain presence of a gene is marked with "+" and the lack of data is highlighted by "?".

A

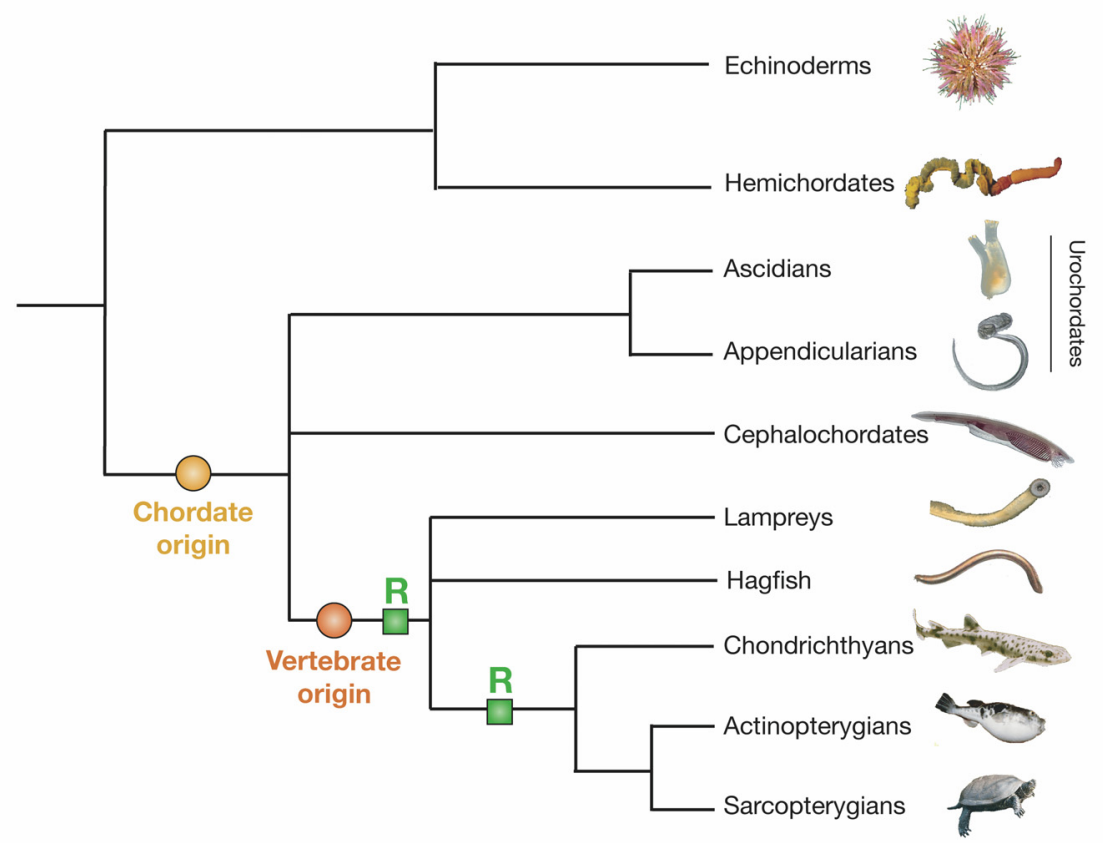

B

WGS RAR Raldh Cyp26

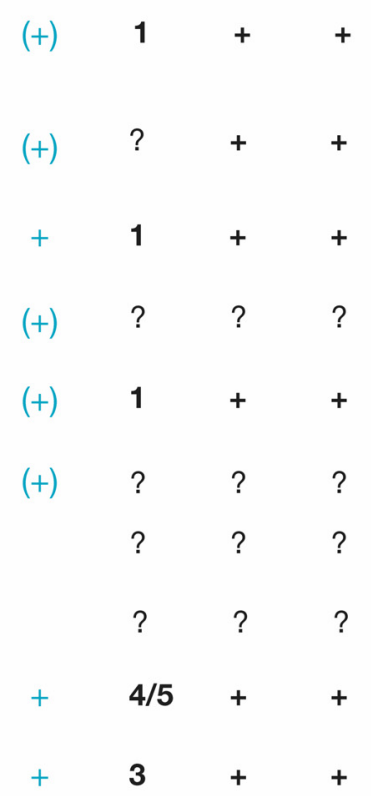

\section{The retinoic acid signaling pathway}

Retinoic acid (RA) is a natural morphogen synthesized from vitamin A (retinol), and it has been known for over fifty years that either too much or too little RA during early development is teratogenic mainly due to anteroposterior patterning defects [3]. In general, excess RA posteriorizes, while RA deficiency anteriorizes chordate embryos [3, 11-14]. Endogenous RA is synthesized in two steps: the first is the reversible oxidation of retinol to retinal performed by alcohol dehydrogenases (ADHs or RDHs/SDRs) and the second is the oxidation of retinal to retinoic acid, which is carried out by retinaldehyde dehydrogenases (RALDHs). Conversely, endogenous RA is degraded by CYP26 enzymes. RA signaling levels are also regulated by binding of retinol to cellular retinol binding proteins (CRBPs) and of RA to retinoic acid binding proteins (CRABPs) (Fig. 2A) [13, 15]. The roles of these proteins in regulating RA signaling in vertebrates have been elucidated with gene knockouts. For example, individual knock-outs of three of the four Raldh genes (i.e. the knock-outs of Raldh1, 2 and 3) have been described in the mouse, but only that of Raldh2 exhibits clear developmental defects. In these mice, the anteroposterior axis is shortened and the embryos, which die before birth, have defects in the heart, limbs and head, which are reminiscent of vitamin A deprivation phenotypes [16]. In the RA degradation pathway, knock-out of Cyp26a1, one of the three mouse Cyp26 genes, is also lethal before birth and affects anteroposterior patterning as well as hindbrain and tail development $[17,18]$. This Cyp26a1 phenotype is comparable to the teratogenic effects of excess RA, suggesting that Cyp26 genes in general 
may help restrict the distribution of endogenous RA to RA target tissues. Moreover, analysis of Cyp26a1 and Raldh2 double knock-out mice has shown that RA and not one of its metabolic derivatives is the major (if not the only) active retinoid during development [19].

Figure 2. Synthesis, degradation and mode of action of retinoic acid (RA). A) The metabolic pathway for synthesis and degradation of endogenous RA is shown. RA is synthesized by oxidation of retinal by retinaldehyde dehydrogenases (RALDHs). In a reversible reaction, retinal is synthesized from retinol (vitamin A) by either aldehyde dehydrogenases (ADHs) or short-chain dehydrogenase/reductases (RDHs/SDRs). Cellular retinol binding proteins (CRBPs) can bind retinol, whereas cellular retinoic acid binding proteins (CRABPs) can bind RA. Finally, endogenous RA is degraded by CYP26 enzymes. B) The RAR/RXR heterodimer mediates the effects of RA. In the absence of ligand (RA), the RAR/RXR heterodimer is bound to DNA and co-repressors. This complex induces transcriptional repression through histone deacetylation. Binding of the ligand (RA) induces conformational changes and the binding of co-activators leading to histone acetylation and activation of transcription.

A

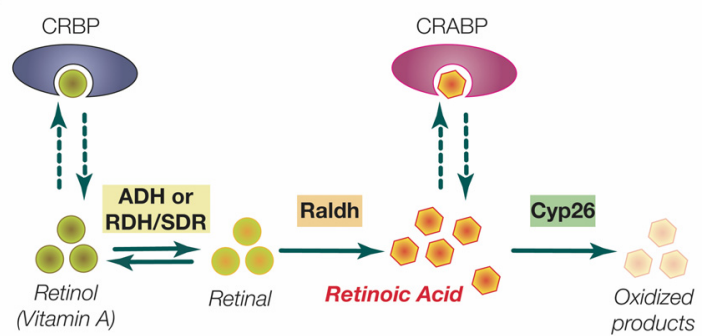

B
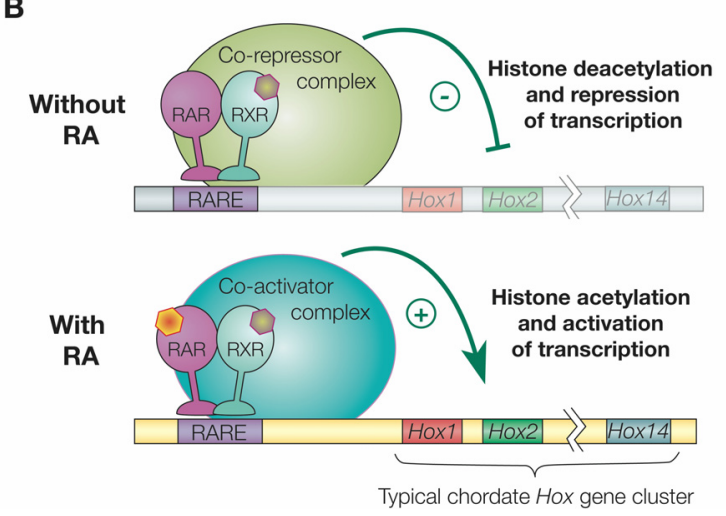

RA signaling is mediated by RA binding to retinoic acid receptors (RARs), which form heterodimers with retinoid $X$ receptors (RXRs). This complex in turn binds to retinoic acid response elements (RAREs) in the regulatory regions of target genes [13, 15]. Only a few direct targets of RA signaling have been described. These include the RARs themselves, Hox genes, some other transcription factors, such as HNF-3a and $C d x 1$, plus genes involved in retinoic acid metabolism (e.g. CRABP1 and 2) [5]. In the mouse, there are 3 RARs and 3 RXRs $(\alpha, \beta$ and $\gamma$ ). Although knock-out of any one of these has only minor, tissue-specific effects on morphogenesis due most likely to functional redundancy among them, compound mutants with two or three of the genes inactivated are much more severely affected with defects in anteroposterior patterning of pharyngeal endoderm, hindbrain and neural crest cells [3].

In general, RAR and RXR proteins share a common organization of functional domains: an amino terminal $\mathrm{A} / \mathrm{B}$ region containing a transcriptional activation domain (AF-1), a centrally located $\mathrm{C}$ region corresponding to the DNA binding domain (DBD) plus a weak dimerization domain and the $E$ region, which includes the ligand binding domain (LBD), a strong dimerization interface and a surface allowing binding of transcriptional coregulators $[3,20,21]$. In the absence of ligand, the RAR/RXR heterodimer is constitutively bound to DNA on RAREs and associated with co-repressor complexes that induce transcriptional silencing by deacetylating histones associated with the target sequences thus increasing chromatin condensation (Fig. 2B). These co-repressors include the related proteins SMRT and NcoR [20-22]. Binding of RA to the RAR ligand binding pocket induces a conformational change of the LBD that creates a surface allowing the association of co-activators and the release of co-repressors. The co-activators (e.g. TIF2 and SRC-1 of the p160 co-activator family) subsequently mediate histone acetylation resulting in decondensation of the chromatin and activation of target gene expression (Fig. 2B) [20-22].

Among the known targets of the RAR/RXR heterodimer are the Hox genes [5]. Hox genes encode transcription factors that contain a highly conserved DNA binding domain of 60 amino acids, the homeodomain $[7,8]$. Hox genes are usually linked in a cluster and their order on the chromosome correlates with both their temporal and spatial expression during embryogenesis [7-9, 23]. This so-called collinear expression is crucial for conveying anteroposterior positional patterning information to the embryo during development and is conserved in many animals, including most deuterostomes [7-9, 23]. However, when genomic Hox clustering has been lost, as for example in tunicates, temporal collinearity is lacking and spatial collinearity is only approximate [24, 25]. In vertebrates, Hox genes are direct targets of RA signaling [5] and RA signaling is involved in regulation of collinear Hox expression along the anteroposterior body axis of the developing embryo. For example, in mice, the deletion of a single RARE from the Hoxa1 cis-regulatory region disrupts the establishment of the anterior boundary of Hoxa1 as well as the normal expression pattern of Hoxa2 in the hindbrain [26]. Moreover, the Hox cluster also contains several other RAREs, which are largely conserved among vertebrates and at least to some extent between vertebrates and the cephalochordate amphioxus $[4,6]$. For example, both the amphioxus and vertebrate Hox 1 genes have a conserved RARE in their cis-regulatory region [4]. In addition, although 
clustering of Hox genes has been lost in tunicates, ascidian Hox 1 expression is strongly upregulated after RA treatment [27] suggesting that, as in amphioxus and vertebrates, Hox genes in tunicates might also be direct targets of RA signaling.

\section{Retinoic acid signaling in invertebrate deuterostomes}

Early during vertebrate diversification, the total number of genes has been markedly increased by two rounds of whole genome duplications [28]. Thus, while vertebrates have 3 or more RARs, there are single RARs in invertebrate chordates, such as amphioxus and tunicates [14, 29]. A comprehensive search of the sea urchin genome sequence (www.ncbi.nlm.nih.gov/genome/guide/sea_urchin) also reveals the presence of a single RAR (Fig. 3). Although less is known about the RA signaling pathway in invertebrate deuterostomes than in vertebrates, it seems likely that the overall pathway is very much the same in amphioxus and vertebrates and somewhat different in tunicates (for example, RAR does not regulate its own expression in tunicates, but it does in both amphioxus and vertebrates [29]). Raldh and Cyp26 genes have been identified in the genomes of both amphioxus (ftp.ncbi.nih.gov/pub/TraceDB/branchiostoma_flori dae) and tunicates (www.ensembl.org/Ciona_intestinalis) (Fig. 1B) [30]. Unfortunately, too little is known about RA signaling in echinoderms, such as sea urchins, for a comparison with chordates [31-33]. Moreover, for hemichordates, the sister group of echinoderms, it is not known if there is an $R A R$, and roles for RA signaling during hemichordate development have yet to be established. Even so, bioinformatic analyses of the sea urchin genome sequence (www.ncbi.nlm.nih.gov/genome/guide/sea_urchin) and of hemichordate EST data (ftp.ncbi.nih.gov/pub/TraceDB/saccoglossus_kowale vskii) suggest that both Raldh and Cyp 26 genes are present in echinoderms and hemichordates (Fig. 1B). Interestingly, these analyses also revealed a putative RARE (a so-called DR5 element) about 3770 base pairs upstream $\left(5^{\prime}\right)$ of the sea urchin Hox1 gene. Thus, at least some components of the RA signaling cascade were probably already present in the last common ancestor of deuterostomes.

Figure 3. Phylogeny of deuterostome retinoic acid receptors (RARs). The tree shows the phylogenetic relationships between RARs from the sea urchin Strongylocentrotus purpuratus, the ascidian tunicate Ciona intestinalis, the cephalochordate Branchiostoma floridae, pufferfish (Takifugu rubripes) and humans (Homo sapiens). The RAR sequences were added to an alignment of nuclear hormone receptors [34] and conserved sites (335 positions) were subsequently selected for phylogenetic reconstruction using PhyML (WAG $+\Gamma_{8}+\mathrm{I}$ ) [61]. 100 bootstrap replicates were carried out to determine the robustness of the obtained phylogenetic tree. In the tree, the RAR subfamily as a whole is strongly supported (bootstrap support 95\%) and within the RARs the sea urchin sequence is at the base (with a moderate support of $70 \%$ ). The respective branching of the cephalochordate and tunicate RARs is not resolved (bootstrap support of 49\%). Nonetheless, the invertebrate chordate sequences are positioned between the sea urchin RAR and the duplicated RARs of vertebrates, which form a single clade that is very strongly supported (100\%). This analysis shows that a $R A R$ gene is present in the genome of echinoderms and suggests that, despite the lack of data from hemichordates, the presence of $R A R$ is an ancestral character of deuterostomes.

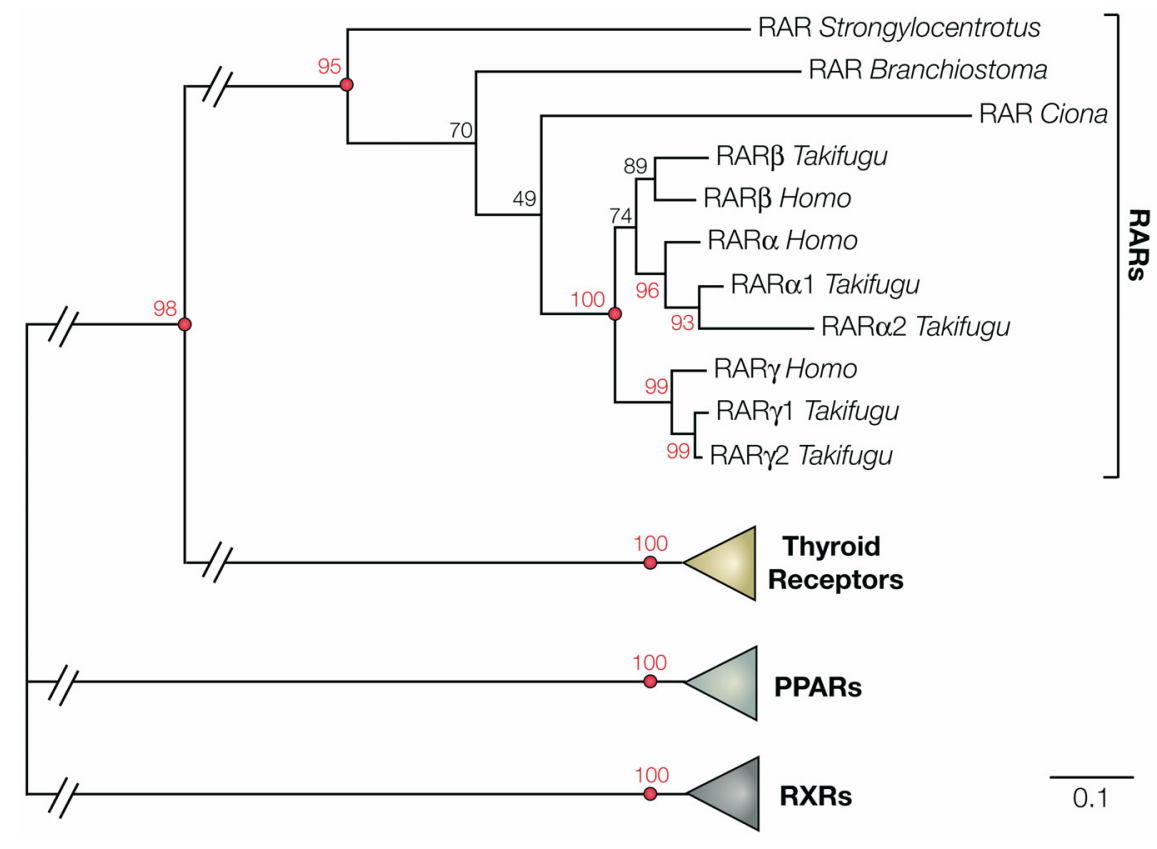

In addition, these results raise two very important questions about the evolution of the RA 
signaling network: (1) If molecular components of the RA pathway are present in non-chordate deuterostomes, what are their functional roles during development? (2) If molecular traces of the RA pathway exist in all deuterostomes, when did RA signaling first evolve? In the last common ancestor of deuterostomes, in the urbilaterian ancestor of deuterostomes and protostomes or even earlier during animal evolution? A first tentative step towards answering the second question was recently taken by an extensive phylogenetic analysis of the nuclear hormone receptor superfamily, which includes the RAR and RXR subfamilies [34]. The results obtained from this analysis suggest that a "Proto-RAR" gene was already present in the genome of the last common ancestor of protostomes and deuterostomes and that in protostomes this RAR gene was lost at least in the lineages leading to nematode worms and insects [34].

\section{The role of RA signaling in chordate morphogenesis}

In vertebrates, the roles of RA signaling in anteroposterior patterning of the central nervous system (CNS) and pharynx have received particular attention $[35,36]$. In the CNS, when vertebrate embryos are treated with RA, anterior neural structures (like the forebrain) are lost and posterior structures, such as the hindbrain and spinal cord, are expanded. In addition, expression of genes in the anterior nervous system (like Otx2) is lost and the Hox genes, normally expressed in hindbrain and spinal cord, are upregulated and shifted anteriorly [35]. In lampreys, for example, RA expands expression of Hox3 anteriorly in the hindbrain [37]. Conversely, decreasing RA signaling levels has the opposite effect: anterior neural structures, such as the forebrain and midbrain are expanded posteriorly, as is expression of Otx2 [35]. Loss of at least two of the three RAR genes in mice also leads to mispatterning of the hindbrain, such as defects in segmental (rhombomeric) hindbrain organization, which correlate with an induction of Hox gene expression at more posterior levels of the hindbrain [38]. Thus, in vertebrates, RA signaling influences collinear expression of Hox genes, which in turn is required to set up an anteroposterior patterning code along the neural tube.

Not only is anteroposterior patterning of the CNS affected by RA signaling, but, since hindbrain neural crest carries the Hox code of the CNS with it when it migrates, the Hox code carried by neural crest migrating into the branchial arches is also altered when levels of RA signaling are changed. In mammals, excess RA causes fusion of the first two pharyngeal arches while in RA-deficient mice and rats, pharyngeal structures caudal to the first pharyngeal arch are absent [39-42]. Similarly in vitamin A-deficient quail, the pharynx is extended caudally, the first pouch forms normally, the second one is abnormal and the third and fourth pharyngeal pouches never form [43]. In lampreys, the effects of excess RA are even more severe, with complete deletion of anterior pharyngeal structures [44]. Thus, RA signaling in the pharynx specifies the anteroposterior position of pharyngeal structures [36, $45,46]$.

It was initially believed that the effects of exogenous RA on the vertebrate pharynx were solely due to a mispatterning of neural crest cells migrating into the branchial arches $[36,45,46]$. However, this influence of neural crest cells is subordinate to regional patterning within the pharyngeal endoderm itself, since removal of neural crest does not prevent the proper patterning of pharyngeal arches and pouches [47]. Conversely, in the zebrafish, $T b x 1$ function in the pharyngeal endoderm is required for proper development of neural crest-derived structures in the pharyngeal arches [48, 49]. Interestingly, the defects obtained in the Tbx 1 mutant van gogh in zebrafish are similar to those obtained after loss of both $R A R \alpha$ and $\beta$ in mice $[38,48,49]$ suggesting that RA signaling may be upstream of Tbx1 in the pharyngeal endoderm. Moreover, treatment of developing mice with an RAR $\beta$-specific agonistic ligand has conclusively shown that RAR $\beta$ dependent RA signaling in the endoderm is independent of neural crest cells [50]. Thus, while neural crest cells migrating into the branchial arches give rise to several structures, such as the pharyngeal arch cartilage, the initial patterning of the pharynx requires RA signaling within the endoderm [36, 45 , 46].

In the cephalochordate amphioxus, $R A R$ is expressed in the hindbrain and anterior spinal cord of the developing CNS [14]. Moreover, in the region of the amphioxus CNS strongly expressing RAR, Hox1, Hox3 and Hox4 are collinearly expressed [51]. RA strongly upregulates $R A R$ expression throughout the CNS, whereas an RA antagonist almost completely downregulates RAR [14]. In addition, RA pushes the anterior limits of Hox1 expression anteriorly in the CNS [12], while RA antagonist shifts the Hox1 domain posteriorly (Fig. 4A) [52]. These data suggest that in cephalochordates, as in vertebrates, RA signaling, probably acting via Hox genes, controls anteroposterior patterning of the developing CNS. Similarly, in the amphioxus general ectoderm, treatments with RA and RA antagonist affect collinear expression of Hox genes. Whereas RA shifts expression of Hox genes (such as Hox1) anteriorly in the general ectoderm, the RA antagonist completely downregulates ectodermal Hox expression (Fig. 4B) [53].

Moreover, in amphioxus, which lacks definitive neural crest, exogenous RA shifts the posterior limit of the pharynx anteriorly, while the application of RA antagonist has the opposite effect $[12,14]$. The amphioxus pharyngeal endoderm strongly expresses Pax1/9 and Otx $[54,55]$ and treatments with RA or an RA antagonist shift the posterior limit of Pax1/9 and Otx expression, respectively, anteriorly or posteriorly [52]. RAR and Hox1 are expressed just posterior to 
Pax1/9 and Otx in the midgut region of the endoderm suggesting that, as in vertebrates, RA signaling within the endoderm might control anteroposterior pharyngeal patterning in amphioxus [52]. In addition, loss of Hox 1 function mimics the effect of a decrease of RA signaling in the amphioxus endoderm [52]. Taken together, these results suggest that in the amphioxus endoderm, RA signaling activates Hox 1 in the midgut and that Hox 1 in turn mediates the effects of RA signaling by limiting expression of genes that are required for specification of the pharynx (such as Pax1/9 and $O t x$ ) to the anterior endoderm [52].

Figure 4. RA signaling controls Hoxl expression in central nervous system (CNS) and general ectoderm of developing amphioxus. A) The rostral limit of Hoxl expression in the CNS (arrowheads) is shifted, respectively, anteriorly and posteriorly by $1 \times 10^{-6} \mathrm{M}$ RA and $1 \times 10^{-6} \mathrm{M}$ RA antagonist (BMS009). Side views of whole mounts of 20 -hour amphioxus embryos with anterior to the left. Scale bar $=50 \mu \mathrm{m}$. " $\mathrm{x}$ " shows the level of the frontal sections in B). B) In the general ectoderm, the rostral limit of $\operatorname{Hoxl}$ (arrowheads) is shifted anteriorly by $1 \times 10^{-6} \mathrm{M}$ RA, whereas treatment with $1 \times 10^{-8} \mathrm{M}$ RA antagonist (BMS009) downregulates Hoxl expression. Frontal sections of 20-hour amphioxus embryos with anterior to the left. Scale bar $=50 \mu \mathrm{m}$. Modified with permission from [52].

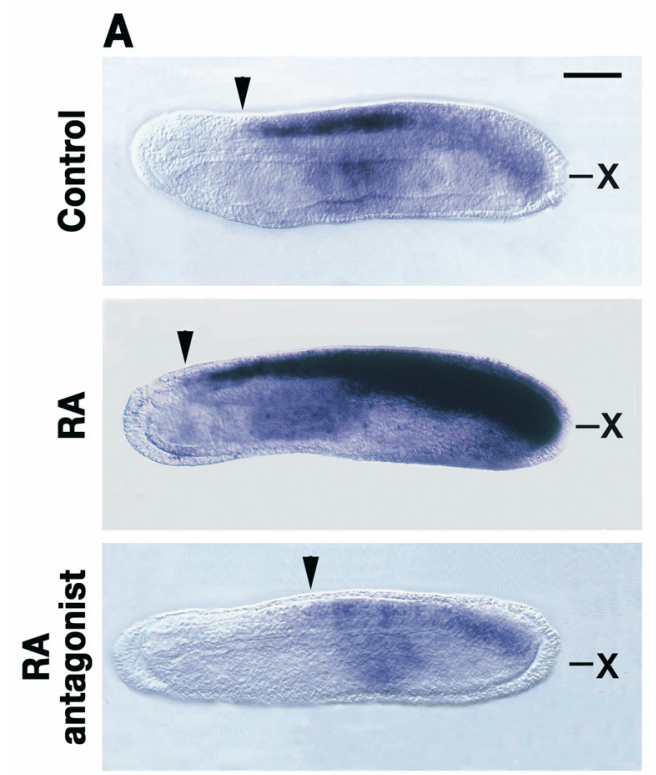

B

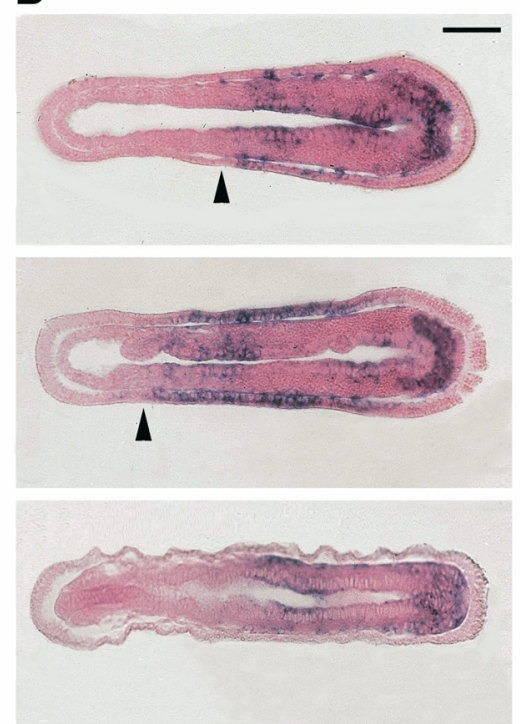

A role for RA signaling and Hox genes in neural patterning of tunicates is much less obvious than in amphioxus and vertebrates. In the ascidian Ciona intestinalis, treatment with RA does not affect the expression of $R A R$ suggesting that, as mentioned above, RAR does not regulate its own expression as it does in other chordate groups [29]. In addition, tunicates have lost several Hox genes and both cluster organization and collinear expression have been at least partly lost. For example, the ascidian Ciona intestinalis has lost Hox7, Hox8, Hox9 and Hox11 [24], while the appendicularian Oikopleura dioica has lost Hox3, Hox6, Hox7 and Hox8 [25]. Expression of some of the remaining Hox genes cannot be detected at all in the CNS and the domains of those that are expressed are only approximately collinear [24, 25]. Even so, excess RA affects morphology and even Hox gene expression at least in ascidian tunicates. For example, treatment of embryos with exogenous RA leads to incomplete closure of the anterior neural tube of Ciona intestinalis [29] and expression of the Hox1 gene in the CNS of the tunicate Halocynthia roretzi is

shifted anteriorly [11]. Thus, a role for RA signaling via Hox genes in neural patterning of tunicates remains a possibility. In contrast to its effects on the CNS, the effects of RA on pharyngeal structures in tunicates is more similar to that in amphioxus and vertebrates. As in amphioxus, Pax1/9 and Otx genes are expressed in the tunicate pharynx during gill slit formation [56, 57]. Moreover, in the ascidian tunicate Herdmania curvata, RA treatment of juveniles leads to a decrease of Otx expression in pharyngeal tissues and to an eventual loss of the pharyngeal basket by respecification of anterior endoderm to a more posterior fate [57, 58]. Taken together, these data suggest that, in tunicates, as in amphioxus, RA signaling might play roles in patterning and development of the CNS and pharyngeal endoderm. In addition, there is evidence that at least some of the genetic machinery mediating RA signaling in amphioxus and vertebrates (like the Hox genes) might also be important for mediating RA signaling in tunicates. Thus, embryonic patterning mechanisms mediated by RA signaling and Hox genes are present in all chordates.

Figure 5. Evolution of RA- and Hox-dependent patterning mechanisms in deuterostomes. In scenario 1, the putative deuterostome ancestor had a central nervous system (CNS) (located ventrally) and both CNS and general ectoderm were patterned by Hox genes, while a role for RA signaling remains elusive. In this scenario, chordates evolved by dorso-ventral axis inversion and the CNS was secondarily lost in the hemichordate lineage. In scenario 2, the nervous system of the 
ancestral deuterostome was organized as an ectodermal nerve net. Hox-dependent patterning codes were present in the ectoderm, but again a role for RA signaling remains elusive. Early during chordate evolution, condensation of a central nervous system (CNS) dorsally led to the creation of two RA-Hox patterning hierarchies one in general ectoderm and one in neural ectoderm (i.e. in the CNS). In the vertebrate lineage, neural crest function was elaborated and neural crest cells contribute to patterning and development of the embryo by carrying positional information from the CNS into other tissues, for example in the pharyngeal region.
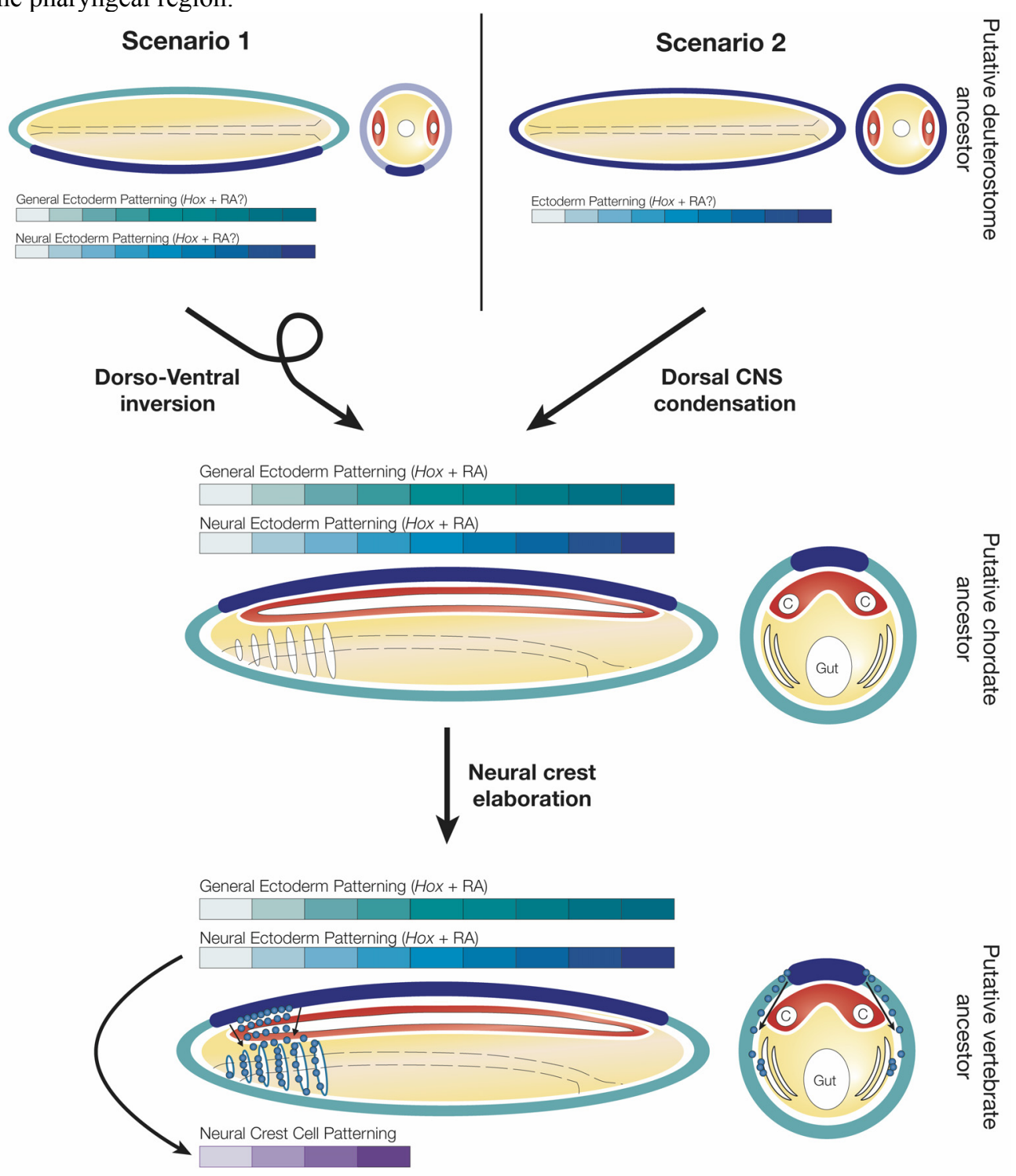

In contrast to chordates, enteropneust hemichordates do not have a centralized nervous system, but instead a diffuse ectodermal nerve net (i.e. a "skin brain") $[59,60]$. As in the cephalochordate amphioxus, Hox genes are collinearly expressed in the hemichordate ectoderm [53, 60]. Whether RA signaling is involved in the control of Hox gene expression in the ectoderm of hemichordates is unknown, but RA does not appear to affect anteroposterior patterning in echinoderms, the sister group of hemichordates [31]. These data suggest that collinear expression of Hox genes was already used for anteroposterior patterning of ectodermal tissues in the last common ancestor of hemichordates and cephalochordates and that a role for RA signaling in controlling collinear expression of Hox genes in the ectoderm evolved very early during chordate evolution. Whether this RA-Hox hierarchy evolved even earlier, in the last common ancestor of all deuterostomes, remains to be determined. Thus, very 
early during chordate evolution or perhaps even very early during deuterostome diversification, RA signaling was co-opted for controlling Hox-dependent anteroposterior patterning mechanisms during development. In one scenario, the putative deuterostome ancestor had a ventral CNS that was patterned by Hox genes like other tissue layers, such as the general ectoderm (Fig. 5), but the CNS was lost in hemichordates [59]. In the second scenario, the ancestral deuterostome lacked a CNS, and the CNS evolved independently from the ectoderm in protostomes and deuterostomes (Fig. 5) [59]. In this scenario, an ancestral ectodermal patterning mechanism was carried over into the CNS at the base of the chordates, leading to the creation of two independent RA-Hox control hierarchies, one in general ectoderm and one in neural ectoderm (i.e. in the CNS) (Fig. 5). Thus, in invertebrate chordates, RA signaling and Hox genes are involved in anteroposterior patterning of the general ectoderm, the CNS, the endoderm (and possibly even of the mesoderm). During vertebrate evolution, with the functional elaboration of neural crest cells another level of complexity has been added. By migrating through the embryo, neural crest cells carry patterning information acquired at the dorsal neural tube into other tissue layers (Fig. 5). In either case, RA signaling became linked to Hox gene function at least at the base of the chordates. Future research on hemichordates will show whether it evolved even earlier in the deuterostome lineage.

\section{Conclusions}

In sum, RA signaling is a key regulator of anteroposterior patterning orchestrated by Hox codes in at least general ectoderm, central nervous system and endoderm of chordates. In contrast to the relative conservation of developmental mechanisms within chordates, evidence for roles of RA outside the chordates and the evolutionary origin of RA signaling itself still remain elusive. In fact, it is unclear when RA-controlled patterning mechanisms first evolved in the animal kingdom and when the RA signaling cascade was co-opted for regulating Hox gene expression. Future research on the evolution of RA signaling should thus focus on the identification of RA signaling components, such as the RA receptor (RAR), in non-chordate deuterostomes (such as hemichordates and echinoderms), protostomes (such as insects and mollusks) and maybe even cnidarians. Furthermore, to fully understand the elaboration of this signaling pathway during evolution, the functional roles of the identified components during development will have to be assessed.

\section{Acknowledgements}

The authors would like to thank Nicholas D. Holland, Cédric Finet, François Bonneton and Yannick Le Parco for helpful comments and critical reading of the manuscript. We are indebted to MENRT, CNRS, ARC and the Région Rhônes-Alpes for financial support. LZH is also supported by NSF grant IOB-0416292 and by MOD grant 1-FY05-108.

\section{Conflict of interest}

The authors have declared that no conflict of interest exists.

\section{Sequence accession numbers}

Strongylocentrotus purpuratus RAR (XM_774883), Ciona intestinalis RAR (AB210661), Branchiostoma floridae RAR (AF378827), Takifugu rubripes RAR $\alpha 1$ (FRUP00000151563), Takifugu rubripes RAR $\alpha 2$ (FRUP00000165122), Takifugu rubripes RAR $\beta$ (FRUP00000079596), Takifugu rubripes RAR 1 (FRUP00000158774), Takifugu rubripes RAR $\gamma 2$ (FRUP00000143282), Homo sapiens RAR $\alpha$ (NM_001033603), Homo sapiens RARß (NM_000965), Homo sapiens RAR $\gamma$ (NM_000966).

\section{References}

1. Rowe T. Chordate phylogeny and development. In: Cracraft J, Donoghue MJ, editors. Assembling the tree of life. New York: Oxford University Press. 2004: 384-409.

2. Gans C, Northcutt RG. Neural crest and the origin of vertebrates: a new head. Science 1983; 220:268-274.

3. Mark M, Ghyselinck NB, Chambon P. Function of retinoid nuclear receptors: lessons from genetic and pharmacological dissections of the retinoic acid signaling pathway during mouse embryogenesis. Annu Rev Pharmacol Toxicol 2006; 46:451-480.

4. Manzanares M, Wada H, Itasaki N, Trainor PA, Krumlauf R, Holland PW. Conservation and elaboration of Hox gene regulation during evolution of the vertebrate head. Nature 2000; 408:854-857.

5. Balmer JE, Blomhoff R. Gene expression regulation by retinoic acid. J Lipid Res 2002; 43:1773-1808.

6. Oosterveen T, Niederreither K, Dolle P, Chambon P, Meijlink F, Deschamps J. Retinoids regulate the anterior expression boundaries of $5^{\prime}$ Hoxb genes in posterior hindbrain. EMBO J 2003; 22:262-269.

7. McGinnis W, Krumlauf R. Homeobox genes and axial patterning. Cell 1992; 68:283-302.

8. Garcia-Fernandez J. The genesis and evolution of homeobox gene clusters. Nat Rev Genet 2005; 6:881-892.

9. Deschamps J, van Nes J. Developmental regulation of the Hox genes during axial morphogenesis in the mouse. Development 2005; 132:2931-2942.

10. Shimeld SM, Holland PW. Vertebrate innovations. Proc Natl Acad Sci USA 2000; 97:4449-4452.

11. Katsuyama Y, Wada S, Yasugi S, Saiga H. Expression of the labial group Hox gene HrHox-1 and its alteration induced by retinoic acid in development of the ascidian Halocynthia roretzi. Development 1995; 121:3197-3205.

12. Holland LZ, Holland ND. Expression of AmphiHox-1 and AmphiPax-1 in amphioxus embryos treated with retinoic acid: insights into evolution and patterning of the chordate nerve cord and pharynx. Development 1996; 122:1829-1838.

13. Ross SA, McCaffery PJ, Drager UC, De Luca LM. Retinoids in embryonal development. Physiol Rev 2000; 80:1021-1054.

14. Escriva H, Holland ND, Gronemeyer H, Laudet V, Holland LZ. The retinoic acid signaling pathway regulates anterior/posterior patterning in the nerve cord and pharynx of amphioxus, a chordate lacking neural crest. Development 2002; 129:2905-2916.

15. Napoli JL. Interactions of retinoid binding proteins and enzymes in retinoid metabolism. Biochim Biophys Acta 1999; 1440:139-162. 
16. Niederreither K, Subbarayan V, Dolle $\mathrm{P}$, Chambon $\mathrm{P}$. Embryonic retinoic acid synthesis is essential for early mouse post-implantation development. Nat Genet 1999; 21:444-448.

17. Abu-Abed S, Dolle P, Metzger D, Beckett B, Chambon P, Petkovich $\mathrm{M}$. The retinoic acid-metabolizing enzyme, CYP26A1, is essential for normal hindbrain patterning, vertebral identity, and development of posterior structures. Genes Dev 2001; 15:226-240.

18. Sakai Y, Meno C, Fujii H, Nishino J, Shiratori H, Saijoh Y, Rossant J, Hamada $\mathrm{H}$. The retinoic acid-inactivating enzyme CYP26 is essential for establishing an uneven distribution of retinoic acid along the anterio-posterior axis within the mouse embryo. Genes Dev 2001; 15:213-225.

19. Niederreither K, Abu-Abed S, Schuhbaur B, Petkovich M, Chambon P, Dolle P. Genetic evidence that oxidative derivatives of retinoic acid are not involved in retinoid signaling during mouse development. Nat Genet 2002; 31:84-88.

20. Laudet V, Gronemeyer H. The nuclear receptor facts book. San Diego: Academic Press, 2001.

21. Gronemeyer H, Gustafsson JA, Laudet V. Principles for modulation of the nuclear receptor superfamily. Nat Rev Drug Discov 2004; 3:950-964.

22. Zechel C. Synthetic retinoids dissociate coactivator binding from corepressor release. J Recept Signal Transduct Res 2002; 22:31-61.

23. Kmita M, Duboule D. Organizing axes in time and space; 25 years of colinear tinkering. Science 2003; 301:331-333.

24. Ikuta T, Yoshida N, Satoh N, Saiga H. Ciona intestinalis Hox gene cluster: its dispersed structure and residual colinear expression in development. Proc Natl Acad Sci USA 2004; 101:15118-15123.

25. Seo HC, Edvardsen RB, Maeland AD, Bjordal M, Jensen MF, Hansen A, Flaat M, Weissenbach J, Lehrach H, Wincker P, Reinhardt R, Chourrout D. Hox cluster disintegration with persistent anteroposterior order of expression in Oikopleura dioica. Nature 2004; 431:67-71.

26. Dupe V, Davenne M, Brocard J, Dolle P, Mark M, Dierich A, Chambon P, Rijli FM. In vivo functional analysis of the Hoxa-1 3' retinoic acid response element (3'RARE). Development 1997; 124:399-410.

27. Ishibashi $T$, Nakazawa $M$, Ono $H$, Satoh $N$, Gojobori $T$, Fujiwara S. Microarray analysis of embryonic retinoic acid target genes in the ascidian Ciona intestinalis. Dev Growth Differ 2003; 45:249-259.

28. Dehal P, Boore JL. Two rounds of whole genome duplication in the ancestral vertebrate. PLoS Biol 2005; 3:e314.

29. Nagatomo K, Ishibashi T, Satou Y, Satoh N, Fujiwara S. Retinoic acid affects gene expression and morphogenesis without upregulating the retinoic acid receptor in the ascidian Ciona intestinalis. Mech Dev 2003; 120:363-372.

30. Nagatomo K, Fujiwara S. Expression of Raldh2, Cyp26 and Hox1 in normal and retinoic acid-treated Ciona intestinalis embryos. Gene Expr Patterns 2003; 3:273-277.

31. Sciarrino S, Matranga V. Effects of retinoic acid and dimethylsulfoxide on the morphogenesis of the sea urchin embryo. Cell Biol Int 1995; 19:675-680.

32. Sconzo G, Fasulo G, Romancino D, Cascino D, Giudice G. Effect of retinoic acid and valproate on sea urchin development. Pharmazie 1996; 51:175-180.

33. Kuno S, Kawamoto M, Okuyama M, Yasumasu I. Outgrowth of pseudopodial cables induced by all-trans retinoic acid in micromere-derived cells isolated from sea urchin embryos. Dev Growth Differ 1999; 41:193-199.

34. Bertrand S, Brunet FG, Escriva H, Parmentier G, Laudet V, Robinson-Rechavi M. Evolutionary genomics of nuclear receptors: from twenty-five ancestral genes to derived endocrine systems. Mol Biol Evol 2004; 21:1923-1937.
35. Maden M. Retinoid signalling in the development of the central nervous system. Nat Rev Neurosci 2002; 3:843-853.

36. Mark M, Ghyselinck NB, Chambon P. Retinoic acid signalling in the development of branchial arches. Curr Opin Genet Dev 2004; 14:591-598.

37. Murakami Y, Pasqualetti M, Takio Y, Hirano S, Rijli FM, Kuratani S. Segmental development of reticulospinal and branchiomotor neurons in lamprey: insights into the evolution of the vertebrate hindbrain. Development 2004; 131:983-995.

38. Dupe V, Ghyselinck NB, Wendling O, Chambon P, Mark M. Key roles of retinoic acid receptors alpha and beta in the patterning of the caudal hindbrain, pharyngeal arches and otocyst in the mouse. Development 1999; 126:5051-5059.

39. Lee YM, Osumi-Yamashita N, Ninomiya Y, Moon CK, Eriksson U, Eto K. Retinoic acid stage-dependently alters the migration pattern and identity of hindbrain neural crest cells. Development 1995; 121:825-837.

40. Wendling O, Dennefeld C, Chambon P, Mark M. Retinoid signaling is essential for patterning the endoderm of the third and fourth pharyngeal arches. Development 2000; 127:15531562.

41. White JC, Highland M, Kaiser M, Clagett-Dame M. Vitamin A deficiency results in the dose-dependent acquisition of anterior character and shortening of the caudal hindbrain of the rat embryo. Dev Biol 2000; 220:263-284.

42. Niederreither K, Vermot J, Le Roux I, Schuhbaur B, Chambon P, Dolle $\mathrm{P}$. The regional pattern of retinoic acid synthesis by RALDH2 is essential for the development of posterior pharyngeal arches and the enteric nervous system. Development 2003; 130:2525-2534.

43. Quinlan R, Gale E, Maden M, Graham A. Deficits in the posterior pharyngeal endoderm in the absence of retinoids. Dev Dyn 2002; 225:54-60.

44. Kuratani S, Ueki T, Hirano S, Aizawa S. Rostral truncation of a cyclostome, Lampetra japonica, induced by all-trans retinoic acid defines the head/trunk interface of the vertebrate body. Dev Dyn 1998; 211:35-51.

45. Graham A, Smith A. Patterning the pharyngeal arches. Bioessays 2001; 23:54-61.

46. Trainor PA, Krumlauf R. Hox genes, neural crest cells and branchial arch patterning. Curr Opin Cell Biol 2001; 13:698-705.

47. Veitch E, Begbie J, Schilling TF, Smith MM, Graham A. Pharyngeal arch patterning in the absence of neural crest. Curr Biol 1999; 9:1481-1484.

48. Piotrowski T, Nusslein-Volhard C. The endoderm plays an important role in patterning the segmented pharyngeal region in zebrafish (Danio rerio). Dev Biol 2000; 225:339-356.

49. Piotrowski T, Ahn DG, Schilling TF, Nair S, Ruvinsky I, Geisler R, Rauch GJ, Haffter P, Zon LI, Zhou Y, Foott H, Dawid IB, Ho RK. The zebrafish van gogh mutation disrupts tbx1, which is involved in the DiGeorge deletion syndrome in humans. Development 2003; 130:5043-5052.

50. Matt N, Ghyselinck NB, Wendling O, Chambon P, Mark M. Retinoic acid-induced developmental defects are mediated by RAR $\beta / R X R$ heterodimers in the pharyngeal endoderm. Development 2003; 130:2083-2093.

51. Wada H, Garcia-Fernandez J, Holland PW. Colinear and segmental expression of amphioxus Hox genes. Dev Biol 1999; 213:131-141.

52. Schubert M, Yu JK, Holland ND, Escriva H, Laudet V, Holland LZ. Retinoic acid signaling acts via Hox1 to establish the posterior limit of the pharynx in the chordate amphioxus. Development 2005; 132:61-73.

53. Schubert M, Holland ND, Escriva H, Holland LZ, Laudet V. Retinoic acid influences anteroposterior positioning of epidermal sensory neurons and their gene expression in a 
developing chordate (amphioxus). Proc Natl Acad Sci USA 2004; 101:10320-10325.

54. Holland ND, Holland LZ, Kozmik Z. An amphioxus Pax gene, AmphiPax-1, expressed in embryonic endoderm, but not in mesoderm: implications for the evolution of class I paired box genes. Mol Mar Biol Biotechnol 1995; 4:206-214.

55. Williams NA, Holland PW. Molecular evolution of the brain of chordates. Brain Behav Evol 1998; 52:177-185.

56. Ogasawara M, Wada H, Peters H, Satoh N. Developmental expression of Pax1/9 genes in urochordate and hemichordate gills: insight into function and evolution of the pharyngeal epithelium. Development 1999; 126:2539-2550.

57. Hinman VF, Degnan BM. Retinoic acid perturbs Otx gene expression in the ascidian pharynx. Dev Genes Evol 2000; 210:129-139.

58. Hinman VF, Degnan BM. Retinoic acid disrupts anterior ectodermal and endodermal development in ascidian larvae and postlarvae. Dev Genes Evol 1998; 208:336-345.

59. Holland ND. Early central nervous system evolution: an era of skin brains? Nat Rev Neurosci 2003; 4:617-627.

60. Lowe CJ, Wu M, Salic A, Evans L, Lander E, Stange-Thomann N, Gruber CE, Gerhart J, Kirschner M. Anteroposterior patterning in hemichordates and the origins of the chordate nervous system. Cell 2003; 113:853-865.

61. Guindon S, Gascuel O. A simple, fast, and accurate algorithm to estimate large phylogenies by maximum likelihood. Syst Biol 2003; 52:696-704.

\section{Author biography}

Ferdinand Marlétaz, B.S., M.A.-I (Ecole Normale Supérieure de Lyon, France), is a graduate student in the Laboratory of Molecular Biology of the Cell at the Ecole Normale Supérieure in Lyon, France. He is currently working on the identification of molecular components of the retinoic acid signaling cascade in invertebrate chordates. He is mainly interested in defining novel characters (morphological or molecular) to improve our current understanding of the phylogenetic relationships between animals.

Linda Z. Holland, B.A., M.A. (Stanford University, Palo Alto, CA, USA), Ph.D. (University of California San Diego, San Diego, CA, USA), is Research Professor in the Marine Biology Research Division, Scripps Institution of Oceanography, University of California San Diego. After receiving her M.A., she worked for fifteen years as a laboratory technician before embarking on her own research on chordate evolution, which led to a Ph.D. and to her current position. She pioneered research on developmental genetics of amphioxus and has been instrumental in the effort to sequence the amphioxus genome. She currently directs a laboratory focused on understanding mechanisms of embryonic patterning in amphioxus as key to understanding how vertebrates evolved from their invertebrate chordate ancestors.

Vincent Laudet, DEA (Université Louis Pasteur, Strasbourg, France), Ph.D. (Molecular Oncology Laboratory, Institut Pasteur, Lille, France), is Professor in the Laboratory of Molecular Biology of the Cell at the Ecole Normale Supérieure in Lyon, France. After his Ph.D., he was recruited as research scientist by the Centre National de la Recherche Scientifique (CNRS) and continued working at the Institut Pasteur in Lille for several years, before being appointed Professor at the Ecole Normale Supérieure in Lyon. He is currently heading a research team working on structure and evolution of nuclear hormone receptors. He is particularly interested in comparative studies on retinoid, steroid and thyroid hormone receptors (using amphioxus, lampreys, zebrafish and mice).

Michael Schubert, Vordiplom (Universität Stuttgart, Germany), Ph.D. (Scripps Institution of Oceanography, University of California San Diego, San Diego, CA, USA), Postdoc (Ecole Normale Supérieure de Lyon, France), is research scientist in the Laboratory of Molecular Biology of the Cell at the Ecole Normale Supérieure in Lyon, France. He is a Fulbright graduate student (San Diego) as well as a DAAD (Lyon) and Marie Curie (Lyon) postdoctoral fellow. In 2004, he was recruited as research scientist by the Centre National de la Recherche Scientifique (CNRS). His current research is mainly focused on the evolution of the retinoic acid signaling cascade in chordates, but more generally he is interested in the evolution of vertebrates from an invertebrate chordate ancestor. 